\title{
El Oficio Divino, fuente de inspiración de los villancicos de Sor Juana
}

S Iguen adelantando los estudios sobre Sor Juana Inés de la Cruz y su obra, pero todavía queda mucho por hacer. Asi, en cuanto a sus villancicos - cuya edición definitiva aún no se hace-, no parece haber otro estudio completo y sistemático que el que de ellos ha hecho don Ezequiel A. Chávez, en su Ensayo de Psicología de Sor Juana Inés de la Cruz. Los estudia a fondo desde el punto de vista psíquico y social; pero hay aspectos más técnicos que no cabían en una obra como la suya: el de las fuentes de los villancicos, por ejemplo. No es éste un punto fácil de analizar, pues los villancicos de Sor Juana son líricos ante todo y por lo tanto sumamente originales. Aun un estudio bastante superficial, sin embargo, revela el hecho de que hay cierta relación entre ellos y la Sagrada Escritura. La vida del santo en cuyo honor fueron compuestos tiene también lugar muy importante, como una de las fuentes de esta clase de composiciones. El punto que este estudio tratará de aclarar es si Sor Juana tomó su inspiración directamente de la Biblia y de la vida del santo como ella la conocía, o a través del Oficio Divino, es decir, de los maitines, de los cuales los villancicos formaban parte extrínseca a fines del siglo xvir.

En primer lugar hay que ver qué eran esos villancicos. Don Alfonso Méndez Plancarte lo ha explicado ya:

El nombre de Villancicos... no conserva en nuestra nomenclatura literaria sino este significado: Canciones de $\mathrm{Na}$ vidad, populares y juntamente exquisitas, cultivadas por Lope, Góngora, Valdivieso... Pero a fines del siglo XVIr, como a lo largo del XVIII, los Villancicos fueton muy otra cosa, así por su importancia social y su amplitud de temas, - regocijo infal- 
table en las mayores fiestas religiosas, no ya sólo en las del Niño Dios-, tanto como por sus dimensiones y su externa distribución.

Los Maitines, la parte del Oficio Divino que con pompa extraordinaria solemnizaba la víspera de los grandes días, se dividen en tres Nocturnos a cada uno de los cuales corresponden tres Salmos. Esta distribución la imitaban los Villancicos de la fiesta, nueve en total y repartidos de a tres en tres en igual número de Nocturnos; y así se entretejian con el canto litúrgico a manera de intermedios o entreactos, donde «todo se volvía donaite y gracia, a la par que confianza, alegría y viva espontaneidad.» 1

En cuanto a su contenido, los villancicos pueden haber tenido cierta semejanza con los salmos y las antífonas o con las lecciones y responsorios, de los cuales cada uno de los tres nocturnos de maitines tiene también tres. La hora canónica de laudes sigue, ordinariamente, a maitines, y no hay razón por que dudar que este haya sido el caso, en los maitines solemnes del tiempo de Sor Juana.

La única manera de determinar si el Oficio Divino influyó en los villancicos de Sor Juana es por medio de un análisis detallado de ambas obras. Para ello se examinarán aquí los once juegos o series de villancicos de esta clase que escribió Sor Juana, tomándolos en orden cronológico, pero agrupando los que tratan de un mismo asunto.

Los primeros villancicos que compuso Sor Juana Inés de la Cruz fueron los que sa cantaron en los maitines de San Pedro Nolasco, en 1677. Tanto estos villancicos como las lecciones del segundo nocturno de maitines están basados en la vida de ese santo. No es posible asegurar, sin embargo, que a Sor Juana le haya venido de aquí su inspiración. Al contrario, es evidente que Sor Juana usó otras fuentes parả escribirlos, pues sus villancicos contienen detalles sobre la vida del santo que no se encuentran en el Oficio Divino. Por ejemplo, dice Sor Juana que San Pedro Nolasco

Dejó su patria, y riquezas,

dejó su noble apellido...2

Por el Oficio Divino sabemos que abandonó su patria, pero no se encuentra allí el detalle de que haya dejado su apellido. Si Sor Juana tiene detalles que no se encuentran en los maitines y no tiene nada 
que solamente hubiera podido venir de esa fuente, es ncesario admitir que no hay pruebas de que ella haya usado el Oficio de San Pedro Nolasco para escribir estos villancicos.

Sor Juana compuso villancicos para que se cantaran en la $\mathrm{Ca}$ tedral de México en la fiesta de la Asunción de la Virgen Maria, en los años de $1679,1685,1687$ y en otro año que no está indicado en la primera edición de sus obras.

Al escribir los villancicos de la Asunción de 1679, Sor Juana sí usó el Oficio Divino de esa fiesta. No es que todos estén inspirados en tal fuente, pero, además de varias reminiscencias aquí y allá, tiene las coplas del último villancico en que traduce partes del Oficio Divino y luego las da en latín.

COPLAS

1. La Madre de Dios Bendita se mira exaltada ya, sobre Angelicales Coros, en el Reino celestial.

Chor. Exaltata est sancta Dei Genitrix super choros Angelorum ad caelestia regna.

1. A1 Cielo subió María, y la turba Angelical, cantando bendice alegre la suprema Magestad.

Chor. Assumpta est Maria in caelum, gaudent Angeli. Laudantes bendicunt Dominum.

1. La Virgen Madre al Etéreo tálamo sube a reinar, a donde en solio de estrellas el Rey de Reyes está.

Chor. Virgo Mater assumpta est ad aethereum thalamum. In quo Rex Regum stellato sedet solio.

1. Hazme digna, Virgen Sacra, para poderte alabar; $y$ contra tus enemigos dame virtud eficaz.

Chor. Dignare me laudare te, Virgo sacrata.

$\mathrm{Da}$ mihi virtutem contra hostes tuos. ${ }^{3}$ 
Sor Juana misma dice de dónde tomó estos versos:

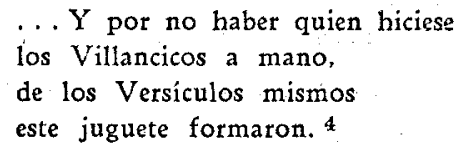

Los hubiera podido tomar de cualquiera de las horas canónicas donde estos mismos versos aparecen como antífonas; pero, si los llama versiculos, ès porque los tomó de maitines donde los tres primeros aparecen como tales, inmediatamente antes de las lecciones del primero, segundo y tercer nocturnos, respectivamente. Los versos que usa para terminar, también se usan como versículos en otros Oficios, pero en el de la Asunción sólo aparecen como la segunda antífona del tercer nocturno. $\mathrm{El}$ único cambio que Sor Juana parece haber introducido, y quizá sea en el Breviario en el que haya ocurrido, es que en vez de comenzar el tercer versículo con las palabras "Maria Virgo", comienza con "Virgo Mater".

En el tercer villancico Sor Juana se refiere al Cantar de los Cantares cuando dice:

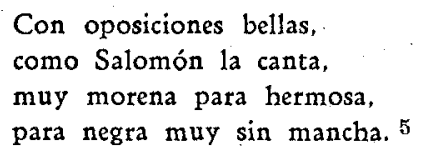

Este pasaje se encuentra en la segunda lección de esos maitines; pero es imposible decir si Sor Juana los tomó de allí o directamente de la Biblia.

Parte de la antífona del himno de laudes se refleja también en estos villancicos. Termina con las palabras "...terríbilis ut castrórum ácies ordináta", 6 y Sor Juana apunta la misma idea, al decir que María es

-.. para combatir muy tierna

para Niña muy armada. 7

En éstos, como en los otros villancicos que escribió Sor Juana, hay numerosas referencias a la Sagrada Escritura; pero como éstas no se' encuentran en los Oficios a que los villancicos corresponden, caen fuera del marco de este estudio. 
Los segundos villancicos de la Asunción (1685) tienen muy poca relación con el Oficio Divino. Quizás estos versos del primer villancico:

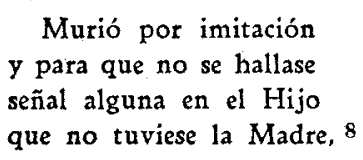

sean una glosa de la quinta lección de estos maitines, en la cual dice San Juan Damasceno que María se sometió a la muerte en obediencia a la antigua sentencia, ya que ni su Hijo se había negado a sufrirla.

Vaga pero interesante es la posibilidad de que la famosa disputa entre las flores y las estrellas, que aparece en el primer villancico del segundo nocturno, haya tenido su inspiración en el Ofício de la Asunción. No es que Sor Juana haya copiado nada ni que se parezcan en detalle, pero tanto el Oficio como la composición de Sor Juana desbordan de lirismo, de astros y de flores. La de Sor Juana comienza con este Estribillo:

Las flores y las estrellas tuvieron una cuestión.

IOh, qué discretas que son, Unas con voz de centellas, y otras con gritos de olores! Oinganlas reñir, señores, que ya dicen sus querellas. 9

Es imposible señalar todas las veces que astros o flores aparecen en los maitines, pues están en todas partes, en el himno, en las antífonas, en las lecciones y en los responsorios. Varias veces se leen estas palabras en maitines:

Et sicut dies verni circúmdabant eam flores rosátum et lília convállium. 10

$\mathrm{Y}$ dicen las flores en los villancicos:

Su fragancia peregrina, más propia la simboliza la Rosa que aromatiza que la Estrella que ilumina. 11 
El sol, la luna, el cielo, las estrellas, todos aparecen en el Oficio de la Asunción. En Sor Juana abundan sobre todo las estrellas.

Estrellas sube a pisar, $y$ en ellas quiere reinar. coronándolas sus huellas. 12

La influencia del Oficio Divino en estos villancicos no es de detalle, sino más bien general: a este respecto, ambas obras tienen el mismo ambiente.

Los villancicos de la Asunción de 1687 incluyen varias referencias a la Biblia. Son muy apropiadas a la fiesta, pero no parecen tener ninguna relación directa con los maitines.

Los villancicos de la Asunción sin fecha, contienen una traducción y comentario de la antífona del himno de laudes del Divino Oficio para esta fiesta:

Quae est ista quae ascéndit sicut auróra consúrgens, pulchra ut luna, elécta ut sol, terríbilis ut castrótum ácies ordináta. 13

He aquí la traducción y el comentario de Sor Juana.

$$
\text { NOCTURNO I }
$$

LETRA III

¿Quién es aquesta hermosura,

Que su salida apresura?

Cual la Aurora presurosa,

$Y$ como la Luna hermosa,

$Y$ como el Sol escogida,

Como Escuadrón guarnecida

De toda fuerte armadura,

¿Quién es aquesta hermosura?

COPLAS

1. ¿Por qué dices, que al Aurora, Se parece su carrera?

2. Porque ella es la luz primera, Que de luz los campos dora. Es del Sol, la precursora, Cuyo divino arrebol 
2. ¿Por qué su beldad sin tasa A Luna, y no a Sol, se encumbra?

1. Porque abrasa el Sol, y alumbra; Pero ella alumbra y no abrasa, $Y$ es luz, que el ardor no pasa, Pues su beldad peregrina,

Sin abrasar ilumina, $Y$ hace favor sin desdén.

Todos. Está bien.

1. Cristo es Sol, que luz propicia

Conserva su Magestad

Entre luces de piedad

Los rayos de la Justicia:

María sólo acaricia,

$Y$ como es sólo Abogada,

Sólo defender le agrada,

$Y$ atender a nuestro bien.

Todos. Está bien.

2. Por eso la Esposa pura

De sus labios celestiales

Sólo destila panales,

Con leche y miel de dulzura:

Mas su Esposo la amargura

Tal vez de Mirra destila,

Porque en sus labios afila,

Cortes de espada también.

Todos. Está bien.

1. Mar digo, ¿por qué razón

Es electa como Apolo?

2. Porque Sol se dijo a Solo.

$Y$ es sola en la perfección.

Una sola en el blasón,

Una sola en la pureza,

Una sola en la belleza,

$Y$ en la dignidad también.

Todos. Está bien.

1. Mas ¿por qué belleza tanta

Es a Escuadrón comparada?

2. Porque está bien ordenada,

$Y$ a todo el infierno espanta:

Cuya vencedora planta,

Quebrantó el cuello orgulloso

De aquel dragón envidioso,

Que cayó con un vainvén

Todos. Está bien, 14 


$$
\begin{aligned}
& \text { Es engendrado del Sol, } \\
& Y \text { es Madre del Sol también. }
\end{aligned}
$$

Todos. Está bien.

Para concluir, se puede decir que en los cuatro villancicos en honor de la Asunción de la Virgen María, Sor Juana tiene pasajes muy hermosos inspirados en el Oficio Divino; pero que, en conjunto, no provienen de esa fuente.

De los villancicos compuestos por Sor Juana para que se cantaran en la Catedral de Puebla de los Angeles, en los maitines de la Purísima Concepción de la Virgen María (8 de diciembre de 1689), no es posible juzgar en estas páginas. La razón es que en tiempo de Sor Juana existían varios Oficios para esa fiesta y sería casi imposible determinar hoy en día para cuál de ellos Sor Juana compuso sus villancicos. Sólo se puede asegurar que no era el mismo que ahora se dice para la fiesta de la Inmaculada Concepción de la Virgen María, pues este fue prescrito por Pío IX en 1863, cuando todos los otros oficios votivos de la Purísima Concepción fueron abolidos.

Dos veces escribió Sor Juana villancicos en honor de San Pedro: los dedicados al Glorioso San Pedro (sin fecha) y los que se cantaron en la Catedral de México en 1683.

Los "Villancicos al Glorioso San Pedro" están llenos de lo que se sabe de este santo por la Sagrada Escritura, y por lo tanto están relacionados, hasta cierto punto, con el Oficio Divino. El primer nocturno comienza con un estribillo puramente lírico. Las coplas que siguen se refieren al Evangelio de la Misa (Mat. xvi, 13-19) y a las lecciones del tercer nocturno que proceden del comentario de San Jerónimo sobre este texto. Sin embargo, Sor Juana es bastante original, concentrándose en el nuevo nombre que Cefas recibe de Cristo.

\footnotetext{
.. Que él es PEDro, responde

Cristo, cuando él Dios vivo le ha llamado, porque tal gloria esconde este nombre de PEDRo venerado, que no hallando a su fe, que satisfaga, sólo en llamarle PEDRo Dios le paga. 15
}

El segundo villancico tiene reminiscencias más bien vagas de la vida $\mathrm{y}$ hechos de San Pedro, mientras que el tercero parece referirse a 
otra parte del mismo Evangelio donde Nuestro Señor promete dar a San Pedro las llaves del Reino de los Cielos.

Contador divino, cuenta, cuenta, cuenta,

y de tu libro borra las deudas nuestras:

$y$ pues tienes en contar

destreza tan singular,

que multiplicas, sumas partes y restas,

multiplica las gracias y parte las penas. 16

El segundo nocturno de los villancicos comienza con versos latinos. Algunos parecen reflejar el sexto responsorio de maitines:

Tu es Pastor óvium, Princeps Apostolótum, tibi trádidit Deus ómnia regna mundi: Et ídeo tibi traditae sunc claves regni caelorum. Quodcúmque ligáveris super terram, erit ligátum et in caelis; et quodcúmque sólveris super terram, erit solútum et in caelis . . 17

Por ejemplo, éstos:

... Qui pastor est animarum,

cui sunt a Christo comissa,

quae Pasqua virentia semper

ovibus ipse distribuat.

Qui Regni caelorum claves

torquet, et aequa iustitia,

quae in terris ligat, et solvit, in caelo solvit, et ligat ... 18

Tanto el resto de este segundo nocturno como el tercero, tratan de varios puntos en la vida de San Pedro; pero no tienen ninguna relación directa con maitines. La herida de Malco en el Jardín de los Olivos, por ejemplo, de que trata el tercer nocturno de los villancicos, está completamente ausente de la liturgia del 29 de junio.

Para concluir se pude decir que las referencias al Oficio Divino son bastante vagas en estos villancicos, y que, aunque hay hechos de que ambas obras tratan, no hay prueba alguna de que Sor Juana los haya usado como fuente de su inspiración.

Los "Villancicos que se cantaron en la Santa Iglesia Catedral de México, en los maitines del Gloriosísimo Príncipe de la Iglesia, 
el Señor San Pedro, año de 1683 ..." son de los más hermosos que escribió Sor Juana. Están llenos de amor, de verdadero lirismo, de sentidas plegarias. No siguen las varias lecciones del Oficio Divino, pues, como dice su autora:

Hoy de PEDRo se cantan las glorias . . 19

Cada villancico trata, no de un hecho de la vida del santo, sino de todas sus glorias, juntando y mezclando todos los hechos históricos. Así, por ejemplo, en el cuarto villancico, en que dice:

Claro Pastor divino, que humildemente grave, quien humilde te mira, soberano te aplaude.

Angular fundamento, en cuyo eterno jaspe asientan de la Iglesia los muros de diamante.

Piedra herida a los golpes del dolor penetrante, desatando tu yelo en dos puros raudales.

Pescador tan dichoso que en un punto te hallaste de dueño de una Barca Piloto de una Nave.

Soberano Clavero de aquellas sacras llaves, que al pecado las cierras, y a la virtud las abres.

Pues tu Sacro Maestro dispuso por honrarte, que $\sin$ tu pasaporte, ninguno al Cielo pase,

\section{Estribitlo}

Duélete de nosotros, Pastor amante, y al ganadillo errante, haz que pase ligero de los pastos humanos, a los eternos. 20 
En el primer villancico trata del diálogo entre Cristo y San Pedro de que hablan el Evangelio de esa fiesto y las lecciones del último nocturno. Pero el asunto más importante de estos villancicos es el llanto de San Pedro, sobre el cual Sor Juana ha sabido escribir hermosísimos versos. Este se halla muy lejos, no sólo de la letra de los maitines, sino hasta del espíritu de esa fiesta de San Pedro. No es nada probable que Sor Juana se haya inspirado en los maitines, para escribir estos villancicos. Tan llena estaba su alma de verdadera devoción a San Pedro, al que alaba y reza espontáneamente, sin necesidad de ningún estímulo externo.

Los Villancicos del Nacimiento de Nuestro Señor Jesucristo (1689) están llenos de regocijo y de ternura; pero no tienen mucha semejanza, ni en tono ni en contenido, con los maities de $\mathrm{Na}$ vidad; a no ser, por supuesto, el asunto de que tratan que es el mismo. Aparecen en ambos el Niño Jesús, la Santísima Virgen, San José, Belén y los pastores. Los villancicos de Sor Juana, sin embargo, no son narrativos sino líricos. Hay dos coincidencias puramente accidentales: el Evangelio de los pastores se encuentra en la octava lección y Sor Juana habla por primera vez de los pastores en el villancico que le corresponde. Al Evangelio que comienza: "In principio erat Verbum..." corresponde la disputa de los sacristanes sobre el "Tantum ergo" y el "Verbum caro", pero estas coincidencias son puramente superficiales.

Los villancicos de San José (1690) contienen una de las pruebas de que existe cierta relación entre el Oficio Divino y los villancicos de Sor Juana. La primera lección del tercer nocturno de maitines comienza con la lectura del Evangelio de San Mateo (I, 18) y sigue una homilia de San Jerónimo. ¿Por qué fué Nuestro Ser̃or concebido de una virgen desposada y no de una libre?, se pregunta el santo, y luego da cuatro razones como respuesta, mencionando que la cuarta es de San Ignacio de Antioquía. El villancico que corresponde a esta lección (primero del tercer nocturno) dice así:

¿Por qué no de simple Virgen,

sino ligada a la unión

del matrimonial consorcio,

el Hijo de Dios nació? 


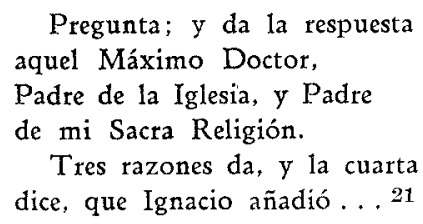

Como puede verse Sor Juana se refiere claramente a la homilia de San Jerónimo dada en la lección de maitines.

Hay partes de estos villancicos que bien pudieran venir del Oficio, pero que también pueden haber venido directamente del Nuevo Testamento. En general, el Oficio Divino y estos villancicos de Sor Juana son muy distintos.

Los últimos villancicos que compuso Sor Juana, y los que contienen algunos de sus más hermosos versos líricos, son los dedicados a Santa Catarina de Alejandría (1691). Don Ezequiel A. Chávez dice que Sor Juana se inspiró, para escribirlos

... en la hermosa leyenda que entonces se tenía universalmente por verdadera historia. 22

No sólo era bien conocida la leyenda, sino que se leía $\rightarrow y$ se lee hoy mismo- en las lecciones de los maitines de que los villancicos formaban parte. En esto los villancicos corresponden muy bien con el Oficio Divino. Cualquier otro detalle que Sor Juana añade, es más bien el resultado de su propio trabajo, que la prueba de que haya usado otras fuentes; sus comparaciones entre Cleopatra y Santa Catarina, por ejemplo. El único detalle que se encuentra en las lecciones de maitines y que Sor Juana no usó, es el que está relatado en la sexta lección. Dice ésta que la esposa de Maximino, el Emperador y el jefe de sus ejércitos fueron a visitar a Santa Catarina en la cárcel y fueron convertidos al cristianismo. Esto en sí no significa que Sor Juana no haya conocido este incidente, sino más bien que no lo quiso usar en una composición que ya estaba tan llena de detalles.

Tanto la Oración de la Misa y del Oficio como los villancicos de Sor Juana, establecen la conexión entre Moisés y Santa Catarina, por ser el Monte Simaí el lugar donde aquél recibió las Tablas de la Ley y ella fue enterrada por los Angeles. 
El oficio de maitines hubiera podido ser fuente suficiente para los villancicos de Santa Catarina; pero esto no significa que Sor Juana lo siga muy de cerca. Cualquier hecho que ella usa no es más que el punto desde donde emprende el vuelo. Por ejemplo, el hecho de que el Emperador Maximino trató de matar a la santa con una rueda de muchas cuchillas, sirve a Sor Juana de punto de partida para versos tan hermosos como los siguientes, en los que mezcla con la historia su propia filosofía.

Contra una tierna rosa, mil cierzos se conjuran. ¡Oh, qué envidiada vive, con ser breve la edad de su hermosura!

Porque es bella la envidian,

Porque es docta la emulan:

¡Oh, qué antiguo en el mundo es regular los méritos por culpas!

De girantes cuchillas En el filo aseguran a un aliento mil soplos, a un solo corazón inmensas puntas.

Contra una sola vida tantas muertes procuran, que es el rencor cobarde, y no se aseguraba bien con una...23

Para concluir, puede decirse que Sor Juana parece haber usado de dos modos, en sus villancicos, los maitines del Oficio Divino: directamente, y como fuente de inspiración. Se refiere directamente al Oficio Divino en los villancicos de la Asunción, sin fecha, y en los de 1679, lo mismo que en los de San José. Hace esto de tres maneras: citando el latín del Oficio Divino y traduciéndolo; traduciéndolo y glosándolo; dando una referencia exacta de lo contenido en una de las lecciones. Como fuente de inspiración Sor Juana parece haber usado el Oficio Divino en los villancicos de la Asunción de 1685, en los del Glorioso San Pedro, y en los de Santa Catarina. Este último uso de los maitines por Sor Juana es naturalmente un punto de conjetura y casi imposible de probar. Ni la misma Sor Juana, quizás, hubiera podido determinar exactamente de dónde tomó los varios materiales que empleó en sus diversas 
composiciones. Basta lo anterior, sin embargo, para poder asegurar que entre las fuentes de los villancicos de Sor Juana deben de considerarse no sólo la Sagrada Escritura y las vidas de los santos, sino también el Oficio Divino.

\author{
Alicia Sarre \\ Barat College of the Sacred Heart \\ Lake Forest, Illinois
}

\title{
$\mathrm{N} O \mathrm{~T} A \mathrm{~S}$
}

1 Alfonso Méndez Plancarte, "Los Villancicos Guadalupanos de Felipe de Santoyo", Abside, II (1938), 18-19.

2 Sor Juana Inés de la Cruz, Poëmas de la Vnica Poetisa Ameticana, Musa Dezima, Sotor Juana Ines de la Cruz... Sacólos a la luz Don Juan Camacho Gayna (Barcelona, 1691, 3a ad.), p. 253.

La ortografía de las poesías de Sor Juana ha sido modificada, para estar de acuerdo con las reglas actuales.

3 Ibid., pp. 249-50.

4 Ibid., p. 249.

5 Ibid., p. 243.

6 Bteviarium Romanum ex Decreto Sactosancti Concilii Tridentini... (Chicago, 1925), Pars Aestiva, p. 989.

7 Sor Juana Inés de la Cruz, Poëmas, p. 244.

8 Ibid., p. 233.

9 Ibid., p. 235.

10 Breviarium Romanum, Pars Aestiva, p. 981.

11 SOR JUANa INÉs de LA CRUZ, Poëmas, p. 236.

12 Ibid., p. 236.

13 Bteviarium Romanum, Pars Aestiva, p. 989.

14 Sor Juana InÉs De la Cruz, Segundo Tomo de las Obras de Sorot Juana Ines de ta Cruz (Barcelona, 1693, $2^{\mathrm{a}}$ ed.), pp. 457-58.

15 SOR JUANA INÉs DE LA CRUz, Poëmas, p. 218.

16 Ibid., p. 220. 
17 Breviarium Romanum, Pars Aestiva, p. 739.

18 SOR JUANA INÉs DE LA CRUZ, Poëmas, pp. 220-1.

19 Ibid., p. 230.

20 lbid., p. 230.

21 lbid., p. 350.

22 Ezequiel A. CHÁvez, Ensayo de Psicología de Sor Juana Inés de la Cruz (Barcelona, 1931), p. 363.

23 SOR JUANA INÉS DE LA CRUZ, Segundo Tomo de las obras de Soror Juana Inés de la Ctuz, p. 70. 
